\title{
Evolution of Broadband Network Management System Using an AOP
}

\author{
EunYoung Cho ${ }^{1}$, Ho-Jin Choi ${ }^{2}$, Jongmoon Baik $^{2}$, In-Young $\mathrm{Ko}^{2}$, \\ and Kwangjoon $\mathrm{Kim}^{1}$ \\ ${ }^{1}$ Electronics and Telecommunications Research Institute, Daejeon, Korea \\ \{eycho,kjk\}@etri.re.kr \\ ${ }^{2}$ Information and Communication University, Daejeon, Korea \\ $\{$ hjchoi,jbaik, iko\}@icu.ac.kr
}

\begin{abstract}
In broadband networks, dynamic service change affects the management system because of the variability in time to market, transmission quality and transport system. Therefore, reusability and maintainability become the important quality factors in Network Management System (NMS). An aspect-oriented software development method is a feasible solution for the evolvability of NMS. In this paper, we propose a method to generate aspects in a standard management information model using Aspect Conversion and Metric Calculation (ACMC) algorithm. We applied it ITU-T M.3100 and evaluated it via the ratio of reduced redundancy in point of crosscutting concerns.
\end{abstract}

\section{Introduction}

In broadband convergence network, various services with Quality of Service (QoS) are required for both customers and service providers. To guarantee reusability and maintainability, there are a number of approaches like Component-Based Software Development (CBSD), Software Product Line (SPL), design pattern. However, in this way, as the systems evolve, crosscutting concerns would be found to be present in legacy object-oriented NMS. This also makes a system difficult to maintain [2]. Therefore, in the presence of not only the new requirements but also evolvability it is necessary to have to meet time-to-market and stability issues. In this paper, we try to present an applicability and benefits of the AOP on the request for change matters as applicable to the NMS.

\section{Aspects in NMS}

In broadband network, quality of service, service level agreement and 99.999\% availability are critical requirements because it is connected to many tributary systems sensitively. Recently, the convergence of circuit and packet transmission is provided in the backbone network for multimedia services. In this environment, NMS takes advantage of the reusability from modularity in AOP [1][3][4]. Figure 1 shows aspects category in each hierarchical layer and network domains. 


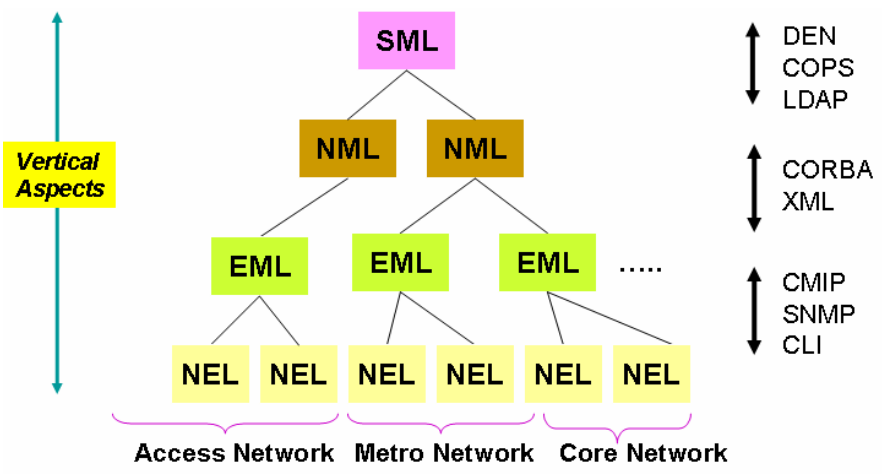

Horizontal Aspects

SML: Service Management Layer DEN Directory Enabled Networks NML: Network Management Layer COPS Common Open Policy Service EML: Element Management Layer LDAP Lightweight Directory Access Protocol NEL: Network Element Layer CMIP Common Management Information Protocol CLI Command Line Interface SNMP Simple Network Management Protocol

Fig. 1. Aspects category in Telecommunications Management Network

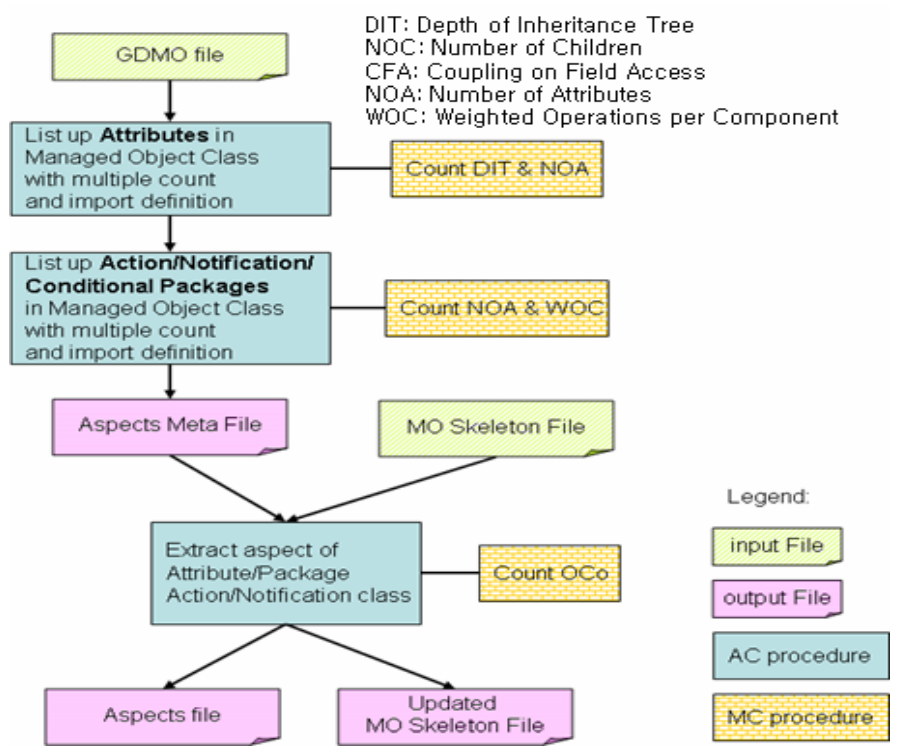

Fig. 2. ACMC Sequence from GDMO definition

\section{Aspect Conversion and Metric Calculation}

Besides logging, transaction, and security concerns, redundant attributes and operations are defined through the standard Management Information Base (MIB). 
Moreover, current management systems operate in the hierarchical layer architecture and various vertical domains. Therefore, aspects are identified as functional aspects within a certain management system and the relationship among network management systems. Figure 2 shows the sequence of ACMC algorithm. The lists of candidate aspects based on key words are generated aspects meta-file. During the generation of aspects, all metrics suite are calculated as an option. At the last step, aspects codes are generated from legacy object-oriented files. If the user wants aspects selectively, it is possible to edit and choose aspects meta-file.

In ITU-T M.3100, there are 36 MOs with 91 conditional packages. After reducing the redundancy, an effectiveness of $48.4 \%$ is obtained.

\begin{tabular}{|l|c|c|l|l|l|l|}
\hline \multicolumn{1}{|c|}{ MO } & Cond. Pkg & DIT & NOC & \multicolumn{1}{|c|}{ CFA } & \multicolumn{1}{|c|}{ NOA } & WOC \\
\hline networkR1 & 1 & 2 & $0 . .4 l$ & 2 & $2+(1)$ & $2 n$ \\
\hline circuitPack & - & 3 & $0 . .1 l$ & 1 & 2 & $3 n$ \\
\hline equipmentR2 & 18 & 2 & $\mathrm{k}$ & 0 & $3+(5)$ & $2 n$ \\
\hline equipmentHolder & 19 & 3 & $2 l$ & 0 & 4 & $4 n+(7 n)$ \\
\hline managedElementR1 & 13 & 2 & $10 l+m$ & $10+(3)$ & $5+(7)$ & $4 n+(2 n)$ \\
\hline $\begin{array}{l}\text { managedElementComple } \\
\text { x }\end{array}$ & 1 & 1 & $0 . .1 l$ & 1 & 2 & $3 n+(2 n)$ \\
\hline softwareR1 & 12 & 2 & $0 . .1 l$ & 1 & $1+(8)$ & $2 n+(17 n)$ \\
\hline CTPBidirectional & 4 & 3 & 0 & $(4)$ & 3 & $5 n+(19 n)$ \\
\hline TTPBidirectional & 15 & 7 & $0 . .1$ & 2 & 2 & $4 n+(12 n)$ \\
\hline Total (9) & 83 & 25 & - & $17+(7)$ & $24+(21)$ & $29 n+(59 n)$ \\
\hline Average & 9.22 & 2.78 & - & $2.1(0.2)$ & $2.7(2.3)$ & $3.2 n(6.6 n)$ \\
\hline
\end{tabular}

$k$ : number of slot at runtime, real number of instances

$l$ : number of children in a specific managed object inherited from managedElement

$m$ : multiple children dependent on transmission capacity

$n:$ : fixed input parameters generated from GDMO compiler

( ): optional or maximum value in case of including conditional packages

Fig. 3. Estimation of reduced ratio in ITU-T M.3100

\section{Conclusion}

This paper shows an application of aspect-oriented software development method in NMS through the application of an algorithm of automatic aspect conversion and metric calculation. It provides for the elimination of redundancy in standard objectoriented management information model and analysis for optimization of the degree of AOP via ACMC algorithm. We contribute to following points: the efficiency increase with the automatic conversion of the aspect-oriented implementation in the object-oriented management information model, the aspect optimization analysis by the automatically generated metric values, the code redundancy reduction, the improvement of reusability and maintainability. 


\section{References}

1. Gandhirajan, A.: Modularizing and Code Reuse Using AOP, http://www.developer.com/ design/article.php/3649681

2. Morris, S.B.: Reducing Upgrade Risk with Aspect Oriented Programming (2005), http://www.onjava.com/pub/a/onjava/2005/03/16/aop-mgmt.html

3. Raz, S.: A Set of Tools to Solve NMS Scaling Using Aspects (2004), http://www.cs.tau. ac.il/ amiramy/SoftwareSeminar/NMSAspect.ppt

4. Cavalli, V., et al.: Report on the availability and characteristics of equipment for nextgeneration networks, IST-2001-34925 (April 2003), http://www.serenate.org/publications/ d9-serenate.pdf 